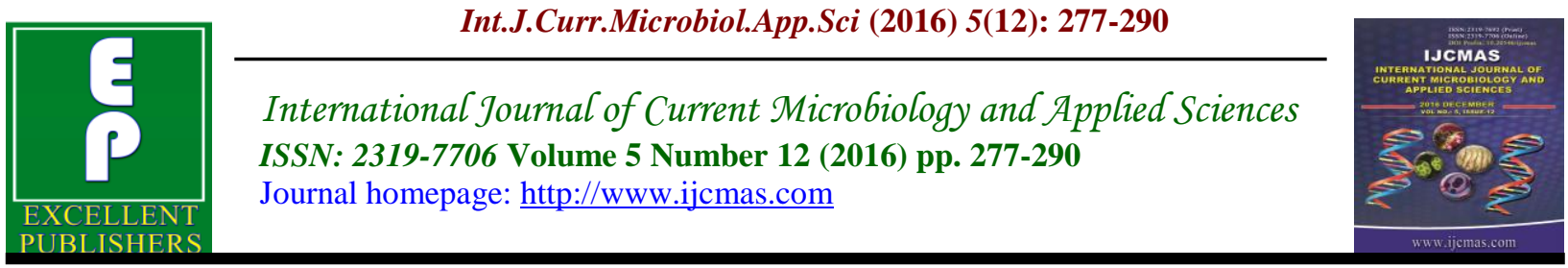

Original Research Article

http://dx.doi.org/10.20546/ijcmas.2016.512.030

\title{
Periodic Surveillance of Systemic Infection Antibiogram a Necessity - A Retrospective and Prospective Study
}

\author{
Shanmuga Vadivoo Natarajan ${ }^{1 *}$, G. Kalaiselvi ${ }^{1}$, B. Usha ${ }^{2}$ and B.K. Padmavathi ${ }^{3}$ \\ Department of Microbiology, Annapoorana medical college \& Hospital, Salem-636308, India \\ *Corresponding author
}

\section{Keywords}

Periodic

Surveillance,

Antibiogram.

\section{Article Info}

Accepted:

12 November 2016

Available Online:

10 December 2016

\section{A B S T R A C T}

Periodic monitoring and surveillance of hospital antibiogram is mandatory because making an Antibiogram is the first step before framing Hospital Antibiotic policy. In this study, during the reference period (Retrospective -January 2013-December 2014 \& Prospective period-January 2015-December 2015) a periodic surveillance of Anatomic site wise stratified antibiogram for blood, wound/soft tissue, respiratory and urine samples was done as per CLSI guidelines. Prevalent rates of Multi Drug resistant (MDR) pathogens-ESBL Enterobactericiae, MRSA, MDR Gram negative Non fermenters were reported. Prevalent Blood stream Pathogens were Klebsiella (17.2\%) \& CONS (27.2\%), Wound pathogens Pseudomonas (25.3\%) \& Staphylococcus aureus (26.4\%), Respiratory tract Pathogens Klebsiella (36.1\%) \& Pseudomonas (22.4\%) and Urinary tract Pathogens E.coli (45\%), \& Klebsiella (17\%). Klebsiella had improved susceptibility for Respiratory \& Blood stream infection when compared to UTI. Pseudomonas showed improved susceptibility profile for both wound and respiratory infection. Drug resistance increased for E.coli during the reference period. Staphylococcus was reported with increasing susceptibility profile when compared with Enterococci. Though there was increasing trend in sensitivity percentage for most of the antibiotics during the prospective period when compared to retrospective period a narrow spectrum of sensitivity was observed for commonly used antibiotics.

\section{Introduction}

In the era of increasing Antimicrobial resistance due to Antimicrobial misuse and reduced emphasis on antibiotic development by pharmaceutical manufacturers. There has been a major international effort to tackle global challenge of Antimicrobial resistance and our national center for disease control has published National treatment guidelines and Antimicrobial policy for antibiotic use in Infectious disease (http://www.ncdc.gov.in/writereaddata/linki mages/AMR_guideline7001495889.pdf). Antibiotic policy is one of the mandatory requirements for accreditation and making an Antibiogram is the first step before framing Antibiotic policy (http://www.ncdc.gov.in/writereaddata/linki mages/AMR_guideline7001495889.pdf; 
Hospital antibiogram, 2010; Infectious Diseases Society of America and the Society for Healthcare Epidemiology of America, 2007). Planning an empiric Antibiotic policy in a hospital utilizes subgroup specific Antibiogram analysis. Standard guidelines for constructing antibiogram are given by organizations like CLSI, IDSA, CDC and WHO (Infectious Diseases Society of America and the Society for Healthcare Epidemiology of America, 2007; Analysis and presentation of cumulative antibiograms, 2007; Specification for a Hospital Cumulative Antibiogram, 2013). They also endorse and recommend the use of appropriate empiric antibiotic therapy based on local microbiological results and their Local Antibiogram. Also most convenient and widely used available measure of a hospital's proportion of resistant organism is hospital cumulative Antibiogram. The need for reliable accurate Antibiogram data is critical to guide appropriate antibiotic selection.

The cumulative hospital Antibiogram is a periodic summary of Antibiotic susceptibility of local bacterial isolates. The objectives of cumulative Antibiogram is to present useful validated information in a consistent way to Clinicians and Policy makers to asses' local susceptibility rates .It also helps in clinician's decision towards selecting appropriate empiric antibiotic therapy and also change in prescribing \& infection control practices. It guides in monitoring antimicrobial resistance trends over time with in an Institute (ICU / ward specific, IP vs. Op) and this can make substantial contribution to patient outcome without additional testing (Atul et al., 2010; Shanmuga et al.,). Aggregates of antibiograms from specific regions would help in monitoring trends across a community \& nation (Diane et al., 2004). Local Antibiogram data is useful for optimized empirical antibiotics therapy.
Antibiograms of pathogens vary markedly between hospitals and between different systemic infection types.

\section{Background}

Susceptibility statistics consisting of cumulative and ongoing summary of patterns of antibiotics sensitivity of clinically important bacteria are important for various health care practitioners \& Microbiologists. The most common use of hospital antibiogram data is probably for assisting clinicians with empiric therapy for suspected infections.

Our tertiary care hospital has been reporting MDR (Multi drug resistant) pathogens from almost all of clinical samples types from different clinical units. As we are in the process of framing an official Antibiotic policy, retrospective surveillance of our hospital Antibiogram will help in framing our Institute's effective Antibiotic policy and prospective surveillance will strengthen the same. This surveillance study will also help in identifying the prevalent MDR pathogens from specific clinical specimen type at our tertiary care center which will ultimately lead to implementation of Specific Infection control practices in those areas accordingly. Moreover, because there will be hardly any new antibiotics in near future, a better understanding of institutional antibiogram is needed on how to optimize the use of existing antibiotics, either alone or in specific combinations.

The aim and objectives of this study includes;

Retrospective Surveillance (Jan 2013-Dec 2014)of Cumulative Antibiogram to identify the most prevalent pathogens isolated from specific anatomic sites \& trends in Antibiotic sensitivity pattern of the same. Prospective Surveillance (Jan 2015-Dec 
2015) of Antibiogram for tracking the shift in trends of MDR pathogens and guidance for a necessity to change in prescribing and infection control practices.

\section{Materials and Methods}

This study was conducted at a 650 bedded tertiary care hospital. All the pathogens isolated from specific clinical samples (Blood, wound/pus, Respiratory tract specimens (throat swabs, sputum, Endotracheal secretions, etc.) and urine)submitted for culture/sensitivity to central Microbiology Lab during the Retrospective period (January 2013December 2014) and Prospective period (January 2015-December 2015) was analyzed. In this study the bacterial profile of Blood stream infections, wound infections, Respiratory tract infections and Urinary tract infections reported. Also the trending antibiogram of the most frequently isolated pathogen of the above mentioned infection syndrome during the mentioned study period was recorded and analyzed. The identification of bacterial isolates and antibiotic susceptibility interpretation for drugs tested against most frequently isolated pathogens is reported according to CLSI guidelines. The cumulative antibiogram was constructed for pathogens isolated from the above mentioned infection syndrome in accordance with the CLSI guidelines. The antibiogram was manually constructed and we included first isolate per patient and excluded duplicate isolates. Trending of Drug resistance /multi drug resistance was recorded forthe prevalent pathogen for the mentioned infection syndrome.

The antibiotic discs that were used to identify the susceptibility pattern of the bacterial pathogens and their concentrations included penicillin $(10 \mathrm{mcg})$, amikacin (30 $\mathrm{mcg}$ ), ceftazidime (70 mcg), ceftriaxone (30 $\mathrm{mcg}$ ), cefoxitin $(10 \mathrm{mcg})$, ciprofloxacin $(5$ $\mathrm{mcg}$ ), clindamycin $(2 \mathrm{mcg})$, erythromycin $(10 \mathrm{mcg})$, gentamicin $(10 \mathrm{mcg})$, imipenem (10 mcg), linezolid (30 $\mathrm{mcg})$, piperacillin/tazobactum(Pip-

Taz)combination (100/10 $\mathrm{mcg})$, and vancomycin $(30 \mathrm{mcg})$.

\section{Results and Discussion}

Antibiotic susceptibility of pathogen can vary markedly between different hospital location and between different clinical specimens. In this study we aim in highlighting the bacterial profile of Blood stream, wound/soft tissue, respiratory tract and Urinary tract infections and cumulative antibiogram of three most prevalent pathogens isolated from each of the above mentioned infection syndrome. Also we have compared the trending susceptibility pattern of the most prevalent isolated pathogen from clinical specimens.

In Table-1 we have enumerated the bacterial profile of Blood stream Pathogens. The most prevalent gram negative being Klebsiella \& Other Nonfermenting Gram Negative Bacilli $(N F G N B)$ and gram positive CONS.

Table-2 highlights the Cumulative sensitivity of most prevalent blood stream pathogens for three year study period (Jan 2013 to Dec 2015)

Trending sensitivity pattern of the prevalent blood stream infection pathogen individually for the each study period is shown in Table3. Last row highlights the Trending drug resistance percentage (ESBL-Extended spectrum beta lactamases) In Table-4 we have enumerated the bacterial profile of Soft tissue/Wound infection. The most prevalent gram negative being Pseudomonas followed by E.coli and gram positive Staphylococcus aureus. 
Table-5 highlights the Cumulative sensitivity of most prevalent wound pathogens for three year study period (Jan 2013 to Dec 2015)

Trending sensitivity pattern of the prevalent wound infection pathogen individually for the each study period is shown in Table-6. Last three rows highlights the Drug resistance percentage (ESBL-Extended spectrum beta lactamases, MRSAMethicillin resistant staphylococcus aureus and MDR \% of Pseudomonas)

In Table-7 we have enumerated the bacterial profile of Respiratory tract Pathogens. The most prevalent gram negative being Klebsiella followed by Pseudomonas and gram positive Staphylococcus aureus.

Table-8 highlights the Cumulative sensitivity of most prevalent Respiratory tract pathogens for three year study period (Jan 2013 to Dec 2015). Trending sensitivity pattern of the prevalent Respiratory pathogen individually for the each study period is shown in Table-9. Last three rows highlights the Drug resistance percentage (ESBL-Extended spectrum beta lactamases, CR-carbapenam resistance, MRSAMethicillin resistant staphylococcus aureus and MDR \% of Pseudomonas)

In Table-10 we have enumerated the bacterial profile of Urinary tract infection. The most prevalent gram negative being E.coli followed by Klebsiella and gram positive Enterococci

Table-11 highlights the Cumulative sensitivity of most prevalent Urinary tract pathogens for three year study period (Jan 2013 to Dec 2015)

Trending sensitivity pattern of the prevalent Uropathogen individually for the each study period is shown in Table-12. Last three rows highlights the Drug resistance percentage
(ESBL-Extended spectrum beta lactamases, CR-carbapenam resistance, VREVancomycin resistant Enterococci)

Cumulative hospital Antibiogram, which is an integral part of Antimicrobial stewardship (AMS) was previously, reported hospital wide. Later CLSI has published consensus guide lines recommending stratification of susceptibility data by patient population wise or Anatomic site wise, or location wise. In this study, during the reference period (Retrospective -January 2013-December 2014 \& Prospective periodJanuary 2015-December 2015) we have done a periodic surveillance of Anatomic site wise stratified antibiogram with specific reference to blood, wound/soft tissue, respiratory and urine.

Blood stream Infection (Table 1, 2 \& Table: 3): For the entire study period the most prevalent gram negatives isolated from blood were Klebsiella (17.2\%) followed by Other NFGNB (13\%) and CONS (27.2\%) among gram positives as shown in Table: 1 . Imipenem and Colistin sensitivity remained $100 \%$ throughout the study period (Table-2 and Table-3)) for both Klebsiella \& Other Nonfermenting Gram negative bacilli. ESBL Klebsiella percentage declined from $100 \%$ in 2013 to $45 \%$ in 2015as shown in Table: 3 .Vancomycin \& Linezolid showed a slight decline in sensitivity for CONS in 2014 but increased to $100 \%$ in 2015 (Table-3). The trending sensitivity for Klebsiella was increasing for most of the antibiotics except Pipericilin-Tazobactum. But for other NFGNB's though Imipenem, Pip-Taz \& Colistin remained $100 \%$ susceptible, there was a declining trend seen for other antibiotics. The susceptibility trend for CONS improved for most of the antibiotics as shown in Table-3

In a study by Shilpi et al., (2016) E.coli was the predominant blood stream pathogen 
(22.4\%) followed by Klebsiella $(19.7 \%)$ and CONS (17.4\%) was the second common among gram positives following Staphylococcus aureus $(18.3 \%)$. In that study Klebsiella showed $100 \%$ sensitivity to colistin but Imipenem sensitivity was 98.2\%.In concordance with our study, reports by Sharma et al., and Pragnya Paramita Jena et al., (2015) CONS was the prevalent gram positive blood pathogen at $21.5 \%$ \& $40.5 \%$ respectively. But Other NFGNB's (22\%) and Acinetobactor (14\%) were the prevalent gram negatives.

Soft tissue/wound infections (Table 4, 5 \& Table: 6): Prevalent gram negative was Pseudomonas (25.3\%) with improving sensitive percentage for ceftazidime from $58 \%$ in 2013 to $70 \%$ in 2015 . Imipenem sensitivity remained $100 \%$ throughout the study period, but colistin\& Pip-taz \% declined in 2015. MDR \% decreased from $41.6 \%$ in 2013 to $13.1 \%$ in 2015 .E.coli second common GNB isolated (14.2\%) showed decline in susceptibility profile in 2015 for most of the antibiotics. Carbapenam resistance increased from 0\% in 2013 to $11 \%$ in 2015 and ESBL percentage also increased from $89 \%$ to $96 \%$. Staphylococcus aureus was prevalent gram positive $(26.4 \%)$ for which Vancomycin \& Linezolid sensitivity improved from $97 \%$ in 2013 to $100 \%$ in 2015 and MRSA rates halved from $40 \%$ in 2013 to $20 \%$ in 2015 . Increasing drug resistance was seen in E.coli.

Similar results are reported by Sah et al., (2013) and dipender et al., (2012) where in Staphylococcus $\quad(41 \% \quad \& \quad 33.8 \%)$, Pseudomonas $(11.5 \%$ \& 20.4\%) \& E.coli $(11.5 \% \& 17.4 \%)$ are predominant wound pathogens respectively .Similar susceptibility profile was reported for Staphylococcus by Sah et al., but improved sensitivity percentage was seen in study by Dipender etal.
Respiratory tract infection (Table 7, 8 \& Table: 9): Prevalent respiratory gram negative pathogen was Klebsiella $(36.1 \%)$ followed by Pseudomonas (22.4\%).Imipenem \& PipericillinTazobactum remained $100 \%$ sensitive for Klebsiella. For Pseudomonas Imipenam sensitivity improved from $97 \%$ in 2013 to $100 \%$ in 2015. Susceptibility profile improved for most antibiotics during the prospective period when compared to retrospective period. MDR percentage for Pseudomonas declined from $10.8 \%$ to $7.1 \%$ and ESBL Klebsiella reduced from $89 \%$ in 2013 to $36 \%$ in 2015. Prevalent Gram positive were Streptococcus (17\%) followed by Staphylococcus aureus (7.0\%).For Staphylococcus Vancomycin \& Linezolid remained $100 \%$ sensitive and MRSA percentage reduced from $21 \%$ in 2013 to $11 \%$ in 2015.

Similar reports from studies by Syed et al., (2013), Sarmah et al., (2016), Vijay et al., (2016) \& Ashok kumar et al., show Klebsiella, Pseudomonas \& Staphylococcus as Predominant respiratory pathogens. Anvari et al., reported Pseudomonas, Acinetobacter as prevalent respiratory pathogens. Syed et al., Anvari et al., \& Vijay et al., reported decreased susceptibility profile for most antibiotics and Sarmah et al., reported a good susceptibility when compared to our study.

Urinary tract infections: The prevalent Gram negative was E.coli $(45 \%)$ followed by Klebsiella (17\%). Imipenem sensitivity reduced for E.coli during the study period (Imipenem 100\% in 2013 \& 94\% in 2015) when compared to Klebsiella (89\% in 2013 $\& 100 \%$ in 2015 ). The sensitivity percentage of other antibiotics like ceftazidime, Norfloxacin \& Nitrofurantoin for E.coli \& Klebsiella remained same at low percentage. ESBL percentage for both E.coli \& Klebsiella almost remained same throughout 
the study period. Enterococci was prevalent gram positive at $13.4 \%$ showed improved sensitivity for Gentamicin \& Nirofurantoin but declining susceptibility profile for Betalactums, Linezolid and Vancomycin.

Table.1 Bacterial profile of Blood stream Infection

\begin{tabular}{|l|l|l|l|l|l|l|l|l|l|}
\hline & & $\mathbf{2 0 1 3}$ & & $\mathbf{2 0 1 4}$ & & $\mathbf{2 0 1 5}$ & \multicolumn{2}{|l|}{ TOTAL } \\
\hline $\begin{array}{l}\text { Total Number } \\
\text { of Samples } \\
\text { received }\end{array}$ & 498 & & 631 & & 1208 & & 2337 \\
& & & & & & & & \multicolumn{2}{|l|}{} \\
\hline & organisms & NO & $\%$ & NO & $\%$ & NO & $\%$ & NO & $\%$ \\
\hline & Klebsiella & 34 & $17 \%$ & 38 & $17.8 \%$ & 40 & $17.2 \%$ & 112 & $\mathbf{1 7 . 2 \%}$ \\
\hline & Salmonella & 21 & $10 \%$ & 25 & $11.7 \%$ & 33 & $14.1 \%$ & 79 & $12 \%$ \\
\hline & E.coli & 20 & $9.5 \%$ & 2 & $0.9 \%$ & 10 & $4.2 \%$ & 32 & $5 \%$ \\
\hline & OtherNFGNB & 17 & $8.0 \%$ & 38 & $17.8 \%$ & 30 & $13 \%$ & 85 & $\mathbf{1 3 \%}$ \\
\hline & Acinetobacter & 22 & $11 \%$ & 8 & $3.6 \%$ & 7 & $3 \%$ & 37 & $6 \%$ \\
\hline & Pseudomonas & 22 & $11 \%$ & 16 & $7.5 \%$ & 21 & $9 \%$ & 59 & $9 \%$ \\
\hline & MSSA & 36 & $17.5 \%$ & 18 & $8.4 \%$ & 12 & $5.2 \%$ & 66 & $10 \%$ \\
\hline & CONS & 33 & $16 \%$ & 68 & $31.8 \%$ & 76 & $33 \%$ & 177 & $\mathbf{2 7 . 2 \%}$ \\
\hline & Enterococci & - & - & 1 & $0.5 \%$ & 3 & $1.3 \%$ & 4 & $0.6 \%$ \\
\hline & & 205 & & 214 & & 232 & & 651 & \\
\hline
\end{tabular}

Table.2 Antibiogram for three year period (Jan 2013 - Dec 2015) of three prevalent Blood Stream Infections (BSI) pathogen Microbiology Data - (n- 651)

\begin{tabular}{|c|c|c|c|}
\hline $\begin{array}{l}\text { Most Common } \\
\text { Pathogens }\end{array}$ & $\begin{array}{l}\text { Number } \\
\text { of Isolate }\end{array}$ & Prevalence \% & Antibiotic Sensitivity \% \\
\hline Klebsiella & 112 & $112 / 651=17.2 \%$ & $\begin{array}{l}\text { Amik-35\%, Genta-13.4\%, } \\
\text { Cefotax-26\%, Ceftaz-21.4\%, } \\
\text { Cipro-0.9\%, Doxy-42\%, Pip taz- } \\
\text { 89.3\%, Imipenem \& Colistin- } \\
\text { 100\%. ESBL: } 78.6 \%\end{array}$ \\
\hline $\begin{array}{l}\text { Other Non-Fermenting } \\
\text { Gram negative bacilli }\end{array}$ & 85 & $85 / 651=13 \%$ & $\begin{array}{l}\text { Amik-65\%,Genta-59\%,Cefotax-49 } \\
\%, \text { Ceftaz-51.\%,Cipro-58\%, } \\
\text { Pip -taz, Imipenem \& Colistin- } \\
100 \%\end{array}$ \\
\hline $\begin{array}{l}\text { Coagulase Negative } \\
\text { Staphylococcus }\end{array}$ & 177 & $177 / 651=27.25 \%$ & $\begin{array}{l}\text { Genta-91\%, Cefazolin-61\%, } \\
\text { Cefotaxime-96\%,Cipro- } \\
\text { 86.4\%,Erythro-79\%,Clinda- } \\
\text { 86\%,Linezolid-99.4\%,Vanco- } \\
\text { 99.4\% }\end{array}$ \\
\hline
\end{tabular}


Table.3 Trending Sensitivity pattern of three prevalent Blood stream Pathogen for three year study period

\begin{tabular}{|l|l|l|l|l|l|l|l|l|l|}
\hline & \multicolumn{3}{|l}{$\begin{array}{l}\text { Klebsiella-17.2\%) } \\
\text { (112-Isolates) }\end{array}$} & \multicolumn{3}{l|}{$\begin{array}{l}\text { NFGNB-13\% } \\
\text { (85-Isolates) }\end{array}$} \\
\hline & 2013 & 2014 & 2015 & 2013 & 2014 & 2015 & 2013 & 2014 & 2015 \\
\hline $\begin{array}{l}\text { No of } \\
\text { isolates }\end{array}$ & $\mathbf{3 4}$ & $\mathbf{3 8}$ & $\mathbf{4 0}$ & $\mathbf{1 7}$ & $\mathbf{3 8}$ & $\mathbf{3 0}$ & $\mathbf{3 3}$ & $\mathbf{6 8}$ & $\mathbf{7 6}$ \\
\hline Amikacin & $11.8 \%$ & $39.5 \%$ & $50 \%$ & $100 \%$ & $39.5 \%$ & $73.3 \%$ & & & \\
\hline Genta & $5.8 \%$ & $21 \%$ & $12.5 \%$ & $94.1 \%$ & $39.5 \%$ & $63.3 \%$ & $100 \%$ & $76.4 \%$ & $100 \%$ \\
\hline Cefazolin & 0 & 0 & $2.5 \%$ & & & & $42.2 \%$ & $90 \%$ & $43.4 \%$ \\
\hline Cefotaxime & $5.8 \%$ & $7.9 \%$ & $60 \%$ & $94.1 \%$ & $60.5 \%$ & $10 \%$ & $100 \%$ & $88.2 \%$ & $100 \%$ \\
\hline Ceftazidime & 0 & $5.3 \%$ & $55 \%$ & $94.1 \%$ & $63.5 \%$ & $10 \%$ & & & \\
\hline Ciprofloxacin & 0 & 0 & $2.5 \%$ & $88.2 \%$ & $60.5 \%$ & $36.6 \%$ & $84.8 \%$ & $88.2 \%$ & $85.5 \%$ \\
\hline Ofloxacin & 0 & $39.5 \%$ & $27.5 \%$ & $94.1 \%$ & $63.5 \%$ & $53.3 \%$ & $81.8 \%$ & $88.2 \%$ & $86.8 \%$ \\
\hline Doxy & $11.8 \%$ & $60.5 \%$ & $40 \%$ & & & & & & \\
\hline Imipenem & $100 \%$ & $100 \%$ & $100 \%$ & $100 \%$ & $100 \%$ & $100 \%$ & & & \\
\hline Pi-Taz & $88 \%$ & $92.1 \%$ & $87.5 \%$ & $100 \%$ & $100 \%$ & $100 \%$ & & & \\
\hline Colistin & $100 \%$ & $100 \%$ & $100 \%$ & $100 \%$ & $100 \%$ & $100 \%$ & & & \\
\hline Erythro & & & & & & & $51.5 \%$ & $78 \%$ & $92.1 \%$ \\
\hline Clinda & & & & & & & $48.4 \%$ & $97 \%$ & $92.1 \%$ \\
\hline Linezolid & & & & & & & $100 \%$ & $98.5 \%$ & $100 \%$ \\
\hline Vanco & & & & & & & $100 \%$ & $98.5 \%$ & $100 \%$ \\
\hline ESBL & $\mathbf{1 0 0 \%}$ & $\mathbf{9 4 . 7 \%}$ & $\mathbf{4 5 \%}$ & NIL & NIL & NIL & & & \\
\hline
\end{tabular}

Table.4 Bacterial profile of Soft tissue/Wound Infection

\begin{tabular}{|c|c|c|c|c|c|c|c|c|c|}
\hline & & 2013 & & 2014 & & 2015 & & \multicolumn{2}{|c|}{ TOTAL } \\
\hline \multirow{2}{*}{$\begin{array}{l}\text { Total Number of } \\
\text { Samples received }\end{array}$} & & 200 & & 238 & & 528 & & \multicolumn{2}{|c|}{966} \\
\hline & Organisms & $\mathrm{NO}$ & $\%$ & $\mathrm{NO}$ & $\%$ & $\mathrm{NO}$ & $\%$ & $\mathrm{NO}$ & $\%$ \\
\hline \multirow{4}{*}{$\begin{array}{l}\text { Gram Negative } \\
\text { bacilli }\end{array}$} & E.coli & 56 & $22 \%$ & 19 & $6 \%$ & 90 & $15.2 \%$ & 165 & $14.1 \%$ \\
\hline & Klebsiella & 20 & $8 \%$ & 13 & $4 \%$ & 33 & $5.6 \%$ & 66 & $5.6 \%$ \\
\hline & Proteus & 12 & $4.8 \%$ & 37 & $11.3 \%$ & 84 & $14.2 \%$ & 133 & $11.3 \%$ \\
\hline & Citrobacter & 4 & $1.6 \%$ & 4 & $1.2 \%$ & 19 & $3.2 \%$ & 27 & $2.3 \%$ \\
\hline \multirow{3}{*}{$\begin{array}{l}\text { Non fermenting } \\
\text { GNB }\end{array}$} & Pseudomo & 48 & $19 \%$ & 76 & $23.2 \%$ & 168 & $28.4 \%$ & 292 & $25.3 \%$ \\
\hline & Other NFGNB & 11 & $4.4 \%$ & 21 & $6.3 \%$ & 13 & $2.2 \%$ & 45 & $3.8 \%$ \\
\hline & Acinetobacter & 10 & $4 \%$ & 4 & $1.2 \%$ & - & - & 14 & $1.2 \%$ \\
\hline \multirow{3}{*}{$\begin{array}{l}\text { Gram positive } \\
\text { cocci }\end{array}$} & Staph aureus & 72 & $28.4 \%$ & 122 & $37 \%$ & 116 & $19.6 \%$ & 310 & $26.4 \%$ \\
\hline & CONS & 12 & $4.8 \%$ & 32 & $9.8 \%$ & 52 & $8.8 \%$ & 96 & $8.2 \%$ \\
\hline & Enterococci & 7 & $3 \%$ & 1 & $0.3 \%$ & 17 & $2.8 \%$ & 25 & $2.1 \%$ \\
\hline TOTAL & & 252 & & 328 & & 592 & & 1172 & \\
\hline
\end{tabular}


Table.5 Antibiogram for three year period (Jan 2013 - Dec 2015) of three prevalent Wound infection pathogen Microbiology Data - (n- 1172)

\begin{tabular}{|c|c|c|c|}
\hline Most Common Pathogens & $\begin{array}{l}\text { Number of } \\
\text { Isolate }\end{array}$ & Prevalence \% & Antibiotic Sensitivity \% \\
\hline Pseudomonas & 292 & $25.3 \%$ & $\begin{array}{l}\text { Amik-84\%,Genta-62\%,Cefotax- } \\
\%, \text { Ceftaz-64.\%,Cipro-67\%, } \\
\text { Pip taz-85.3\%, Imipenem \& } \\
\text { Colistin-100\%. MDR-30.1\% }\end{array}$ \\
\hline E.coli & 165 & $14.2 \%$ & $\begin{array}{l}\text { Amik-88\%,Genta-38\%,Cefotax- } \\
\text { 8\%,Ceftaz-10\%,Cipro-13.3\%,Doxy- } \\
\text { 13.3\%,Pip taz-82\%, Imipenem - } \\
\text { 94\%\& Colistin-99\% } \\
\text { ESBL-90.3\% }\end{array}$ \\
\hline Staphylococcus aureus & 310 & $26.4 \%$ & $\begin{array}{l}\text { Genta-68.3\%, Cefazolin-64\%, } \\
\text { Cefotaxime-71\%,Cepoxitin-73 } \\
\text { 5Cipro-46\%,Erythro-63.2\%,Clinda- } \\
\text { 78\%,Linezolid-99.4\%,Vanco-98.7\% } \\
\text { MRSA-27\% }\end{array}$ \\
\hline
\end{tabular}

Table.6 Trending Sensitivity pattern of three prevalent wound infection Pathogen for three year study period

\begin{tabular}{|c|c|c|c|c|c|c|c|c|c|}
\hline & \multicolumn{3}{|c|}{$\begin{array}{l}\text { Pseudomonas-25.3\% } \\
\text { (292-Isolates) }\end{array}$} & \multicolumn{3}{|c|}{$\begin{array}{l}\text { E.coli-14.2\% } \\
\text { (165-Isolates) }\end{array}$} & \multicolumn{3}{|c|}{$\begin{array}{c}\text { Staphylococcus-26.4\% } \\
\text { (310-Isolates) }\end{array}$} \\
\hline & 2013 & 2014 & 2015 & 2013 & 2014 & 2015 & 2013 & 2014 & 2015 \\
\hline No of isolates & 48 & 76 & 168 & 56 & 19 & 90 & 72 & 122 & 116 \\
\hline Amikacin & $75 \%$ & $68 \%$ & $92 \%$ & $67 \%$ & $89.4 \%$ & $100 \%$ & & & \\
\hline Genta & $41.6 \%$ & $59.2 \%$ & $69 \%$ & $29 \%$ & $42.1 \%$ & $42.2 \%$ & $56 \%$ & $67.2 \%$ & $78 \%$ \\
\hline Cefazolin & - & & - & $7 \%$ & $10.5 \%$ & $4.4 \%$ & $56 \%$ & $57 \%$ & $43.1 \%$ \\
\hline Cefotax & - & & - & $11 \%$ & $15.7 \%$ & $4.4 \%$ & $78 \%$ & $62.3 \%$ & $56.8 \%$ \\
\hline Ceftazidime & $58 \%$ & $56.5 \%$ & $69 \%$ & $11 \%$ & $31.5 \%$ & $4.4 \%$ & & & \\
\hline Cipro & $58 \%$ & $67.1 \%$ & $69 \%$ & $7 \%$ & $31.5 \%$ & $13.3 \%$ & $13.8 \%$ & $46 \%$ & $67.2 \%$ \\
\hline Oflox & $75 \%$ & $70.4 \%$ & $70.2 \%$ & $7 \%$ & $42.1 \%$ & $15.5 \%$ & $16.6 \%$ & $51 \%$ & $78 \%$ \\
\hline Doxy & - & - & - & $14 \%$ & $21 \%$ & $22 \%$ & $35 \%$ & $66 \%$ & $91.4 \%$ \\
\hline Imipenem & $100 \%$ & $100 \%$ & $100 \%$ & $100 \%$ & $100 \%$ & $88.8 \%$ & & & \\
\hline Pi-Taz & $75 \%$ & $100 \%$ & $82 \%$ & $75 \%$ & $89 \%$ & $78 \%$ & & & \\
\hline Colistin & $83.3 \%$ & $100 \%$ & $91.6 \%$ & $100 \%$ & $100 \%$ & $98 \%$ & & & \\
\hline Erythro & & & & & & & $56 \%$ & $64 \%$ & $67.2 \%$ \\
\hline Clinda & & & & & & & $67 \%$ & $85.2 \%$ & $78 \%$ \\
\hline Linezolid & & & & & & & $97 \%$ & $100 \%$ & $100 \%$ \\
\hline Vanco & & & & & & & $97 \%$ & $95 \%$ & $100 \%$ \\
\hline MDR & $41.6 \%$ & $34.2 \%$ & $13.1 \%$ & & & & & & \\
\hline ESBL & & & & $89 \%$ & $68 \%$ & $96 \%$ & & & \\
\hline MRSA & & & & & & & $40 \%$ & $25 \%$ & $20 \%$ \\
\hline
\end{tabular}


Table.7 Bacterial profile of Respiratory Tract Infection

\begin{tabular}{|l|l|l|l|l|l|l|l|l|l|}
\hline & & 2013 & & 2014 & & 2015 & \multicolumn{2}{|c|}{ TOTAL } \\
\hline $\begin{array}{l}\text { Total Number of } \\
\text { Samples received }\end{array}$ & 429 & & 659 & & 1310 & & 2398 \\
\hline \multirow{5}{*}{ Gram Negative bacilli } & Organisms & NO & $\%$ & NO & $\%$ & NO & $\%$ & No & $\%$ \\
\cline { 2 - 12 } & Klebsiella & 56 & $26.3 \%$ & 72 & $30.5 \%$ & 211 & $43.2 \%$ & 339 & $36.1 \%$ \\
\cline { 2 - 11 } & E.coli & 10 & $4.7 \%$ & 9 & $3.8 \%$ & 9 & $1.8 \%$ & 28 & $2.9 \%$ \\
\hline & citrobacter & 1 & $0.5 \%$ & - & - & 9 & $1.8 \%$ & 10 & $1.1 \%$ \\
\hline Non fermenting GNB & Pseudomonas & 36 & $17 \%$ & 47 & $20 \%$ & 127 & $26.0 \%$ & 210 & $22.4 \%$ \\
\cline { 2 - 11 } & Acinetobact & 40 & $18.7 \%$ & 23 & $9.7 \%$ & 9 & $1.8 \%$ & 72 & $7.7 \%$ \\
\cline { 2 - 11 } & $\begin{array}{l}\text { Other } \\
\text { NFGNB }\end{array}$ & 11 & $5.2 \%$ & 27 & $11.4 \%$ & 34 & $7.0 \%$ & 72 & $7.7 \%$ \\
\hline Gram positive cocci & Staph aureus & 30 & $14 \%$ & 24 & $10.2 \%$ & 18 & $3.7 \%$ & 72 & $7.7 \%$ \\
\cline { 2 - 11 } & CONS & 3 & $1.3 \%$ & 6 & $2.5 \%$ & 9 & $1.8 \%$ & 18 & $2.0 \%$ \\
\cline { 2 - 10 } & Enterococci & 2 & $1 \%$ & 1 & $0.5 \%$ & 4 & $0.8 \%$ & 7 & $0.7 \%$ \\
\cline { 2 - 10 } & Streptococci & 24 & $11.3 \%$ & 27 & $11.4 \%$ & 59 & $12.1 \%$ & 110 & $11.7 \%$ \\
\hline TOTAL & & 213 & & 236 & & 489 & & 938 & \\
\hline
\end{tabular}

Table.8 Antibiogram for three year period

(Jan 2013 - Dec 2015) of three prevalent Respiratory tract Infection pathogen Microbiology Data - (n- 938)

\begin{tabular}{|c|c|c|c|}
\hline $\begin{array}{l}\text { Most Common } \\
\text { Pathogens }\end{array}$ & $\begin{array}{l}\text { Number of } \\
\text { Isolate }\end{array}$ & Prevalence \% & Antibiotic Sensitivity \% \\
\hline Klebsiella & 339 & $36.1 \%$ & $\begin{array}{l}\text { Amik-95\%,Genta-63.\%,Cefotax } \\
\text { 53\%,Ceftaz-50\%,Cipro-76\%,Doxy- } \\
\text { 68\%,Pip taz-100\%, Imipenem- } \\
\text { 98.5\% Colistin-100\% } \\
\text { ESBL-50\% }\end{array}$ \\
\hline Pseudomonas & 210 & $22.4 \%$ & $\begin{array}{l}\text { Amik-99\%,Genta-88\%,Ceftaz - } \\
\text { 90\%,Cipro-90\%, } \\
\text { Pip taz-98\%, Imipenem -98.5\%\& } \\
\text { Colistin-99.5\%,MDR-10.5\% }\end{array}$ \\
\hline Staphylococcus aureus & 72 & $7.7 \%$ & $\begin{array}{l}\text { Genta-83\%, Cefazolin-74\%, } \\
\text { Cefotaxime-86\%,,Cipro- } \\
\text { 57\%,Erythro-73.6\%,Clinda- } \\
\text { 89\%,Linezolid-100\%,Vanco-100\% } \\
\text { MRSA-17\% }\end{array}$ \\
\hline
\end{tabular}


Table.9 Trending Sensitivity pattern of three prevalent Respiratory Pathogen for three year study period

\begin{tabular}{|c|c|c|c|c|c|c|c|c|c|}
\hline & \multicolumn{3}{|c|}{$\begin{array}{l}\text { Klebsiella-36.1\% } \\
\text { (339-isolates) }\end{array}$} & \multicolumn{3}{|c|}{$\begin{array}{l}\text { Pseudomonas-22.4\% } \\
\text { (210- isolates) }\end{array}$} & \multicolumn{3}{|c|}{$\begin{array}{l}\text { Staphylococcus-7.7\% } \\
\text { (72- isolates) }\end{array}$} \\
\hline & 2013 & 2014 & 2015 & 2013 & 2014 & 2015 & 2013 & 2014 & 2015 \\
\hline $\begin{array}{l}\text { No of } \\
\text { isolates }\end{array}$ & 56 & 72 & 211 & 36 & 47 & 127 & 30 & 24 & 18 \\
\hline Amikacin & $96.4 \%$ & $90 \%$ & $96.1 \%$ & $100 \%$ & $96 \%$ & $100 \%$ & & & \\
\hline Genta & $89.3 \%$ & $60 \%$ & $56.2 \%$ & $81 \%$ & $85 \%$ & $91 \%$ & $60 \%$ & $100 \%$ & $100 \%$ \\
\hline Cefazolin & & & & & & & $\mathbf{5 0} \%$ & $91.6 \%$ & $89 \%$ \\
\hline Cefotax & $14.3 \%$ & $50.3 \%$ & $64.4 \%$ & $86 \%$ & $85 \%$ & 93\% & $80 \%$ & $91.6 \%$ & $89 \%$ \\
\hline ceftazidime & $11 \%$ & $37 \%$ & $64.2 \%$ & $86 \%$ & $85 \%$ & $93 \%$ & & & \\
\hline Cipro & $80.3 \%$ & $60 \%$ & $80 \%$ & $81 \%$ & $85 \%$ & $94 \%$ & $\mathbf{5 0} \%$ & $50 \%$ & $78 \%$ \\
\hline Doxy & $45 \%$ & $50.3 \%$ & $68.2 \%$ & & & & $\mathbf{5 0} \%$ & $66.6 \%$ & $89 \%$ \\
\hline Imepene & $100 \%$ & $97.2 \% \%$ & $100 \%$ & $97.2 \%$ & $95.7 \%$ & $100 \%$ & $100 \%$ & $100 \%$ & $100 \%$ \\
\hline Piepricillin & & & & $89 \%$ & $87.2 \%$ & $87 \%$ & & & \\
\hline Pi-Taz & $100 \%$ & $100 \%$ & $100 \%$ & $94.2 \%$ & $95.7 \%$ & $100 \%$ & & & \\
\hline Colistin & $100 \%$ & $100 \%$ & $100 \%$ & $100 \%$ & $97.8 \%$ & $100 \%$ & & & \\
\hline Erythro & & & & & & & $\mathbf{5 0} \%$ & $91.6 \%$ & $89 \%$ \\
\hline Clinda & & & & & & & $80 \%$ & $100 \%$ & $89 \%$ \\
\hline Linezolid & & & & & & & $100 \%$ & $100 \%$ & $100 \%$ \\
\hline Vanco & & & & & & & $100 \%$ & $100 \%$ & $100 \%$ \\
\hline MRSA \% & & & & & & & $21 \%$ & $17 \%$ & $11 \%$ \\
\hline ESBL \% & $89 \%$ & $63 \% /$ & $36 \% /$ & - & - & - & & & \\
\hline MDR & & & & $10.8 \%$ & $17.1 \%$ & $7.1 \%$ & & & \\
\hline
\end{tabular}

Table.10 Bacterial profile of Urinary Tract Infection

\begin{tabular}{|l|l|l|l|l|l|l|l|l|l|}
\hline & & 2013 & & 2014 & & 2015 & & \multicolumn{2}{l|}{ TOTAL } \\
\hline $\begin{array}{l}\text { Total Number of Samples } \\
\text { received }\end{array}$ & & 929 & & 1377 & & 2636 & & \multicolumn{2}{l|}{4942} \\
\hline \multirow{5}{*}{ Gram Negative bacilli } & Organisms & NO & $\%$ & NO & $\%$ & NO & $\%$ & NO & $\%$ \\
& E.coli & 139 & $34.8 \%$ & 211 & $35.5 \%$ & 600 & $54 \%$ & 950 & $45 \%$ \\
\cline { 2 - 11 } & Klebsiella & 82 & $20.4 \%$ & 129 & $21.7 \%$ & 147 & $13.2 \%$ & 358 & $17 \%$ \\
\cline { 2 - 11 } & Proteus & 9 & $2.6 \%$ & 22 & $3.6 \%$ & 28 & $2.5 \%$ & 59 & $2.8 \%$ \\
\cline { 2 - 11 } & Citrobacte & 17 & $4 \%$ & 33 & $5.6 \%$ & 19 & $1.7 \%$ & 69 & $3.3 \%$ \\
\hline \multirow{5}{*}{ Non fermenting GNB } & Pseudo & 43 & $10.8 \%$ & 33 & $5.6 \%$ & 56 & $5 \%$ & 132 & $6.3 \%$ \\
\cline { 2 - 11 } & Acineto & 4 & $1.1 \%$ & 11 & $1.9 \%$ & - & - & 15 & $0.7 \%$ \\
\cline { 2 - 11 } & Other NFGNB & - & - & - & - & 38 & $3.4 \%$ & 38 & $1.8 \%$ \\
\hline \multirow{5}{*}{ Gram positive cocci } & Staph & 1 & $0.3 \%$ & 0 & - & 28 & $2.5 \%$ & 29 & $1.4 \%$ \\
\cline { 2 - 11 } & CONS & 65 & $16.2 \%$ & 44 & $7.4 \%$ & 66 & $6 \%$ & 175 & $8.3 \%$ \\
\cline { 2 - 10 } & Enterococci & 39 & $9.8 \%$ & 111 & $18.7 \%$ & 130 & $11.7 \%$ & 280 & $13.4 \%$ \\
\hline TOTAL & & 399 & & 594 & & 1112 & & 2105 & \\
\hline
\end{tabular}


Table.11 Antibiogram for three year period

(Jan 2013 - Dec 2015) of three prevalent Urinary tract Infection pathogen Microbiology Data (n- 2105)

\begin{tabular}{|c|c|c|c|}
\hline Most Common Pathogens & $\begin{array}{l}\text { Number of } \\
\text { Isolate }\end{array}$ & Prevalence \% & Antibiotic Sensitivity \% \\
\hline E.coli & 950 & $45 \%$ & $\begin{array}{l}\text { Amik-84.4\%,Genta-35.3\%,Ceftaz-25.7\%, } \\
\text { Ceftrioxone-24.3\%,Norflox-22 } \\
\%, \text { Nitrofurantoin-93.2\%, Cotrimox-26.3\%, Pip } \\
\text { taz-92.5\%, Imipenem -97.5\%\& Colistin-100\% } \\
\text { ESBL-74.3\% }\end{array}$ \\
\hline Klebsiella & 358 & $17 \%$ & $\begin{array}{l}\text { Amik-82 \%,Genta-34\%,Ceftaz-23.2 \%, } \\
\text { Ceftrioxone-25.1\%,Norflox-31.3 } \\
\% \text {,Nitrofurantoin-72\%, Cotrimox-35 \%, Pip taz- } \\
100 \% \text {, Imipenem -97.2\%\& Colistin-99.4\% } \\
\text { ESBL-76.8\% }\end{array}$ \\
\hline Enterococci & 280 & $13.4 \%$ & $\begin{array}{l}\text { Pen-15.4\%, Amp-27.5\%,Cefazolin- } \\
\text { 38.2\%,Norflox-33.9\%,Nitrofurantoin- } \\
\text { 36.4\%,Linezolid-97.9\%,HCG-48.9\%, vanco- } \\
\text { 98.9\%\% } \\
\text { VRE-1.1\% }\end{array}$ \\
\hline
\end{tabular}

Table.12 Trending Sensitivity pattern of three prevalent Urinary Tract infection pathogen for three year study period

\begin{tabular}{|c|c|c|c|c|c|c|c|c|c|}
\hline & \multicolumn{3}{|c|}{$\begin{array}{l}\text { E.coli }-45 \% \\
\text { (950-isolates) }\end{array}$} & \multicolumn{3}{|c|}{$\begin{array}{l}\text { Klebsiella }-17 \% \\
\text { (358 -isolates) }\end{array}$} & \multicolumn{3}{|c|}{$\begin{array}{l}\text { Enterococci-13.4\% } \\
\text { (280-isolates) }\end{array}$} \\
\hline & 2013 & 2014 & 2015 & 2013 & 2014 & 2015 & 2013 & 2014 & 2015 \\
\hline No of isolated & 139 & 211 & 600 & 82 & 129 & 147 & 39 & 111 & 130 \\
\hline Amikacin & $81 \%$ & $90 \%$ & $90 \%$ & $59 \%$ & $78 \%$ & $100 \%$ & & & \\
\hline Genta/HCG & $41 \%$ & $29 \%$ & $37 \%$ & $63 \%$ & $30 \%$ & $20 \%$ & $23 \%$ & $30 \%$ & $73 \%$ \\
\hline cefazolin & & & & & & & $70 \%$ & $28 \%$ & $50 \%$ \\
\hline Ceftriox & $7 \%$ & $42 \%$ & $22 \%$ & $22 \%$ & $32 \%$ & $19.7 \%$ & & & \\
\hline ceftaz & $12 \%$ & $42 \%$ & $23 \%$ & $22 \%$ & $27 \%$ & $20.4 \%$ & & & \\
\hline Norflox & $16 \%$ & $24 \%$ & $22 \%$ & $22 \%$ & $27 \%$ & $20.4 \%$ & $34 \%$ & $35 \%$ & $33 \%$ \\
\hline Nitrofuran & $84 \%$ & $97 \%$ & $94 \%$ & $48 \%$ & $78 \%$ & $40.1 \%$ & $23 \%$ & $25 \%$ & $50 \%$ \\
\hline Cotrimoxazol & $19 \%$ & $29 \%$ & $27 \%$ & $32 \%$ & $30 \%$ & $10.2 \%$ & & & \\
\hline Imepene & $100 \%$ & $100 \%$ & $96 \%$ & $89 \%$ & $100 \%$ & $100 \%$ & & & \\
\hline \multicolumn{10}{|l|}{ Piepricillin } \\
\hline Pip-Taz & $81 \%$ & $90 \%$ & $96 \%$ & $100 \%$ & $100 \%$ & $100 \%$ & & & \\
\hline Colistin & $100 \%$ & $100 \%$ & $100 \%$ & $97 \%$ & $100 \%$ & $100 \%$ & & & \\
\hline Linezolid & & & & & & & $100 \%$ & $100 \%$ & $95.4 \%$ \\
\hline Vanco & & & & & & & $100 \%$ & $99 \%$ & $98.5 \%$ \\
\hline$\overline{\text { ESBL \% }}$ & $78 \%$ & $58 \%$ & $77 \%$ & $78 \%$ & $73 \%$ & $80 \%$ & & & \\
\hline
\end{tabular}


Studies by Eswarappa et al., (2011) Aswani et al., (2014) Verma et al., (2016) Syed et al., (2012) \& Lathika et al., (2015) reported E.coli \& Klebsiella as predominant Uropathogens. Aswani et al., showed enterococci as prevalent gram positive pathogen. All the studies except Lathika et $a l$, reported good susceptibility profile for common antibiotics tested for UTI when compared to our study.

During the reference period overall there was a decrease in prevalence of drug resistant pathogens irrespective of specimen type. Vancomycin resistant Enterococci decreased from $6 \%$ in 2013 to $1.2 \%$ in 2015,MRSA reduced from $41 \%$ in 2013 to $20 \%$ in 2015, ESBL Klebsiella pneumonia from $89 \%$ in 2013 to $51 \%$ in 2015, Carbapenam resistant Klebsiella reduced from $4.7 \%$ to $0.5 \%$, MDR Acinetobacter from $52 \%$ in 2013 to $25 \%$ in 2015, Carbapenam resistant Acinetobacter from $28 \%$ to $25 \%$,MDR Pseudomonas from $37 \%$ to $12 \%$, carbapenam resistant Pseudomonas from $2.4 \%$ in 2013 to $1.8 \%$ in 2015.Carbapenam resistant E.coli increased from $0 \%$ to $4.7 \%$. With reference to individual prevalent pathogens, there was a low susceptibility profile for E.coli for both wound infections and UTI. Klebsiella had better susceptibility profile for respiratory infections when compared to blood stream infections \& UTI. Pseudomonas showed improved susceptibility profile for Respiratory infection when compared to wound infections. Staphylococcus aureus showed better susceptibility profile for both wound \& respiratory infections.

In conclusion, during the reference period though, there was increase in sensitivity percentage for most of the antibiotics during the prospective period when compared to retrospective period a narrow spectrum of sensitivity was observed for commonly used antibiotics. An empirical antimicrobial Guideline was drafted following retrospective antibiogram Surveillance. Following retrospective period an educational intervention with specific reference to Infection control measures \& antimicrobial stewardship had proven to be modestly effective in our study.

\section{References}

Amy, L., Pakyz. 2007. The Utility of Hospital Antibiograms as Tools for Guiding Empiric Therapy and Tracking Resistance Insights from the Society of Infectious Diseases Pharmacists. Pharmaco-Ther., 27(9): 1306-1312.

Ashok kumar, Kingston rajiah, Chandrasekhar, S. 2012. Antibiotics surveillance: A survey on the susceptibility of microorganisms to antibiotics in respiratory tract infections. Int. J. Pharmacy and Pharmaceutical Sci., Vol 4, Issue 4.

Aswani, S.M., Chandrashekar, U., Shivashankara, K., Pruthvi, B. 2014. Clinical profile of urinary tract infections in diabetics and nondiabetics. The Australasian Med. J., 7(1): 29-34 doi:10.4066/AMJ.2014.1906.

Atul, K., Patel, Ketan, K., Patel, Kamlesh, R., Patel, Sanjiv Shah, Pratibha Dileep. 2010. Time Trends in the Epidemiology of Microbial Infections at a Tertiary Care Center in West India. Over Last 5 Years. (C) Supplement To Japi, vol. 58, 37-58.

Delhi- PragnyaParamita Jena et al. 2015. Int. J. Biomed. Res., 6(10): 819-824.

Diane, C., Halstead, Noel Gomez and Yvette, S., McCarter. 2004. Reality of Developing a Community-Wide Antibiogram. J. Clin. Microbiol., 42: p. $1-6$. 
Eshwarappa, M., Dosegowda, R., Aprameya IV, Khan MW, Kumar PS, Kempegowda P. Clinicomicrobiological profile of urinary tract infection in south India. Indian $J$. Nephrol., 21(1): 30-36.

Gupta, S., Kashyap, B. 2016. Bacteriological profile and antibiogram of blood culture isolates from a tertiary care hospital of North India. Trop. J. Med. Res., 19: 94-9.

Hindler, J.F., Stelling. 2007. Analysis and presentation of cumulative antibiograms: a new consensus guideline from the Clinical and Laboratory Standards Institute. J. Clin. Infect. Dis., 44(6): 867-73.

Hospital antibiogram. 2010. A Necessity, Indian J. Med. Microbial., 28(4): 27780.

http://www.ncdc.gov.in/writereaddata/linki mages/AMR_guideline7001495889.pd $\mathrm{f}$

Infectious Diseases Society of America and the Society for Healthcare Epidemiology of America Guidelines for Developing an Institutional Program to Enhance Antimicrobial Stewardship. 2007. Clin. Infect. Dis., 44(2): 159-177.

KaurNajotra, Dipender, Kakru, Dalip, K. 2012. Bacteriology and antibiogram of skin and soft tissue infections from a tertiary care hospital. Indian J. Med. Specialities, vol. 3 Issue 1, p26

Latika, J., Shah, Geeta, M., Vaghela, Hetvi Mahida. 2015. Urinary tract infection: bacteriological profile and its antibiotic susceptibility in western india. NJMR, 5: 71-74.

Maryam Sotoudeh Anvari et al. 2014. Microbiologic Spectrum and Antibiotic Susceptibility Pattern among Patients with Urinary and Respiratory Tract Infection. Int. J. Microbiol.
Sah, P., Khanal, R., Upadhaya, S. 2013. Skin and soft tissue infections: bacteriological profile and antibiotic resistance pattern of isolates. $J$. Universal College of Med. Sci., Vol.1 No.03.

Sarmah, N., Sarmah, A., Das, D.K. 2016. A Study on the Microbiological Profile of Respiratory Tract Infection (RTI) in Patients Attending Gauhati Medical College \& Hospital. Ann. Int. Med. Den. Res., 2(5): MB11-MB15.

Shanmuga Vadivoo, N., M.D. Sharda, D. Rewa, Kolukula Sujatha, Mahalingam Niranjana, Bavani Manivannan, Nemani, V.K., Sridevi. Antibiogram Analysis and Altering Antimicrobial Susceptibility Pattern of Multidrug Resistant Pathogens. GJMR, Volume 14 Issue 4.Page 23-33.

Sharma, R., Sharma, S. Gupta. 201. Bacteriological analysis of blood culture isolates with their antibiogram from a tertiary care hospital. IJPSR, Vol. 6(11): 4847-4851.

Specification for a Hospital Cumulative Antibiogram. 2013. Australian Commission on Safety and Quality in Health Care. site: http://www.safetyandquality.gov.au/pu blications-resources/publications/

Sunil Vijay, Gaura, V., Dalela. 2016. Prevalence of LRTI in Patients Presenting with Productive Cough and Their Antibiotic Resistance Pattern. $J$. Clin. Diag. Res., Vol-10(1): 9-12.

Syed Mustaq Ahmed et al., 2012. Urinary Tract Infections - An overview on the Prevalence and the Anti-biogram of Gram Negative Uropathogens in A Tertiary Care Centre in North Kerala, India. J. Clin. Diag. Res., Vol-6(7): 1192-1195.

Syed mustaqahmed, Rama krishnapaiJakribettu, Shaniyakoyakuttymeletath, Arya, B., 
Shakirvpa. 2013. Lower Respiratory Tract Infections (LTRIs): An Insight into the Prevalence and the Antibiogram of the Gram Negative, Respiratory, Bacterial Agents. J. Clin. Diag. Res., Vol-7(2): 253-256.

Verma Devki, Mohan Sneha and Chander Yogesh. 2016. Antibiogram Profile of Uropathogens in a Tertiary Care
Hospital in Western Uttar Pradesh, India. Int. J. Curr. Microbiol. App. Sci., 5(6): 422-430.

Zapantis et al. 2005. Nationwide Antibiogram Analysis Using NCCLS M39-Guidelines. J. Clin. Microbiol., p. 2629-2634.

\section{How to cite this article:}

Shanmuga Vadivoo Natarajan, G. Kalaiselvi, B. Usha and B.K. Padmavathi. 2016. Periodic Surveillance of Systemic Infection Antibiogram a Necessity -A Retrospective and Prospective Study. Int.J.Curr.Microbiol.App.Sci. 5(12): 277-290. doi: http://dx.doi.org/10.20546/ijcmas.2016.512.030 